\title{
Modelling of Implantable Sensor Packaging based on Biocompatible Polymers
}

\author{
M. Cacciola ${ }^{1}$, A. Lay-Ekuakille ${ }^{2}$, A. Massaro ${ }^{3}$, F. Spano ${ }^{3}$, D. Caratelli ${ }^{4}$, F.C. Morabito ${ }^{1}$, R. Cingolani ${ }^{3}$, \\ A. Athanassiou ${ }^{3}$, A. Trotta ${ }^{5}$ \\ ${ }^{1}$ University "Mediterranea" of Reggio Calabria, DIMET, 89060 Reggio Calabria (RC), Italy \\ matteo.cacciola@unirc.it \\ ${ }^{2}$ Dipartimento di Ingegneria dell'Innovazione, University of Salento, 73100 Lecce, Italy \\ ${ }^{3}$ Center of Bio-Molecular Nanotechnology, Italian Institute of Technology,73010 Arnesano (Le), Italy \\ ${ }^{4}$ Delft University of Technology, IRCTR, Mekelweg 4, 2628 CD Delft, The Netherlands \\ ${ }^{5}$ Dipartimento di Elettrotecnica ed Elettronica, Polytechnic of Bari, 70100 Bari Italy
}

\begin{abstract}
Implantable devices are being used for long term healthcare and human body physiological monitoring of specific parameters. PDMS (poly-dimethyl-siloxane) can be used either for sensor or for packaging of the same sensor in many applications, notably in laparoscopy and laparotomy as feeler pin in order to help surgeons to touch specific tissues and to get a response based on the nature of the touched tissue (softness). The need of calibrated pressure on a tissue arises when we are in presence particular pathologies or impairments as: cancer issues, high risk of aneurism for aorta and brain, probable haemorrhage in touching capillaries, and so forth. This paper presents a packaging modelling of a build nanosensor to be used in a human body for surgery exploration as laparoscopy and laparotomy. A nanosensor is first build for common application and adapted for implantable applications, and a packaging is studied. The designed sensor is implemented by considering PDMS as polymeric material.
\end{abstract}

Keywords - Biomedical device, Implantable nanodevice, nanosensor packaging, Modelling and Reliability, polydimethylsiloxane and chitosan, Laparoscopy and Laparotomy.

\section{INTRODUCTION}

Advances in microelectronics, microelectrode array and signal processing have enabled neuroscientists and researchers in biomedical engineering to achieve fully implantable devices. Biomedical implantable devices have been an important research issues and are widely considered as key topics for better understanding, controlling and eventually restoring neurological and physiological functions using implantable microsystems. This has made possible to develop a variety of implantable bio-system applications such as treating spinal cord injuries, deep brain stimulation to treat Parkinson's disease and detection of epilepsy [1]-[6].

The packaging technology for the aforementioned applications must be mechanically durable and chemically stable in the physiological environment. For while operating implant lifetimes of several months to a few years are sufficient in chronic animal research, human prostheses may require operating lifetimes that extend over decades. Cables and their connection to integrated sensors must survive several hundred million bending movements, since the human heart beats on the order of 10 million times per year.

Yet, while these mechanical and chemical requirements are not to be neglected, they are not special to integrated devices. The same requirements have been satisfied for years by conventional cardiac pacing leads. The special problem in packaging implantable integrated device is preventing corrosion. Note that this is equivalent to preventing ionic leakage currents. In cardiac pacemakers, wires to pacing electrodes do not carry DC potentials. For these and other implanted stimulation and biopotential telemetry systems which place no DC potentials on electrode leads, the operating $\mathrm{pH}$-potential conditions are within the corrosion immunity regions of the alloys and noble metals which have been chosen for use as implanted cable conductors. Integrated sensors, however, require several diode-drops of voltage to be present on cables continuously. These pH-potential conditions are outside the corrosion immunity regions of all the metals used in conventional cardiac pacing leads. If these metals are exposed to physiological fluid under these conditions, an electrolytic cell will be set up corroding and eventually breaking the more positively biased conductors.

The encapsulation of implanted circuitry has been reviewed or discussed several times in the past. Boretos [7] discussed "those aspects of encapsulation that have consistently caused the greatest degree of difficulty with electronic implants" and surveyed "the materials and methods that produce the best results". Jeutter [8] summarized the encapsulation of discrete components and pointed out some of the problems assembling and packaging hybrid microcircuits. Donaldson [9] pointed out the need for hardshell encapsulation of hybrid microcircuits, and other authors described the hard-shell encapsulation of hybrid microcircuits in detail, including the basic requirements of packaging materials and the performance of those materials which have been used to date.

\section{POLYMER-BASED DEVICE AND PROPOSED MODELLING}

The proposed poly-dimethyl-siloxane (PDMS) sensor [10], as illustrated in Fig. 1, can be obtained by means of a simple fabrication process. The surface of the cantilever will be functionalized in order to trap the biological target. The different weights due to the targets will allow the deflection of the cantilever beam as illustrated in Fig. 2. Since our aims and possible applications of the PDMS pressure sensor, we numerically evaluated the mechanical toughness and 
resilience of the sensor, and the ability to corrosion standing of the packaging. We exploited a commercial software package, Comsol Multiphysics ${ }^{\circledR}$, able to provide a simulating and implementing framework in which different physical phenomena can be combined in so called multiphysical models.

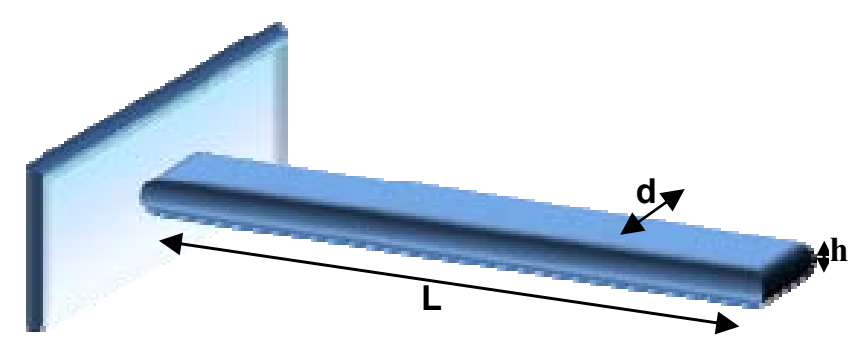

Fig. 1. PDMS micro-cantilever .
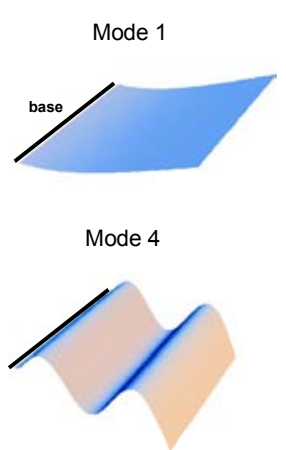
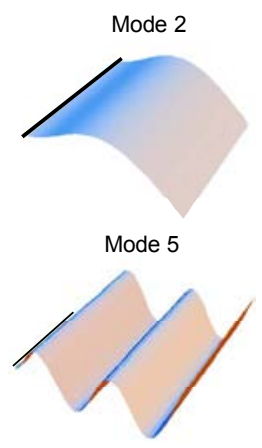
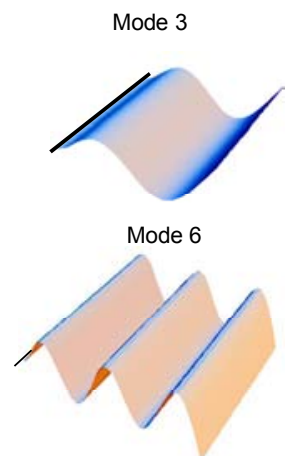

Fig. 2. First six mechanical modal profile of the microcantilever sensor.

Depositing of different layers composing the surface of the sensor can cause localized cracking and micro-scale voids, which could affect the performances of the sensor. In this case, it is important to evaluate the internal residual stresses between the layers during the deposition of materials, which can affect the integrity of sensor. We assumed that the residual stresses in the plate are mostly due to the deposition of the first layer, which is directly in contact with the plate. We implemented the time-dependent Goldak's semi ellipsoidal moving heat source Equation (1) [11] in an axial symmetric numerical model, exploiting the symmetry of the geometry, boundary conditions and loads with respect to the YZ plane, and thus saving CPU time:

$$
Q(x, y, z, t)=\frac{6 \sqrt{3} q_{0}}{a b c \pi \sqrt{\pi}} \exp \left(-\frac{3 x^{2}}{a}-\frac{3 y^{2}}{b}-\frac{3(z-v t)^{2}}{c}\right)
$$

where: $a, b, c$ are the parameters of the ellipsoid; $q_{0}$ is obtained by a calibration so that the temperature of deposition is in the range of the melting temperature of gold (it is a function of the mass rate of deposited metal), and finally $v$ is the speed of the movement during the deposition process. The symmetry plane is thermally insulated. Moreover, we assumed that there is no convection between the plate and the external atmosphere. For the mechanical boundary conditions, the aim is to prevent rigid body motion of the plate, and be as close as possible to the manufacturing process. We know that the plate is subjected to bending due to the high difference of temperature between the plate and the deposited layer. We also know that the plate, during the manufacturing, is clamped to prevent this phenomenon. In Fig. 3, Von Mises stresses are shown. We can notice an increase of the stresses near the boundaries. We do not take this value in account since its high value is only due to boundary effects. More details will be provided in the full version of the paper.

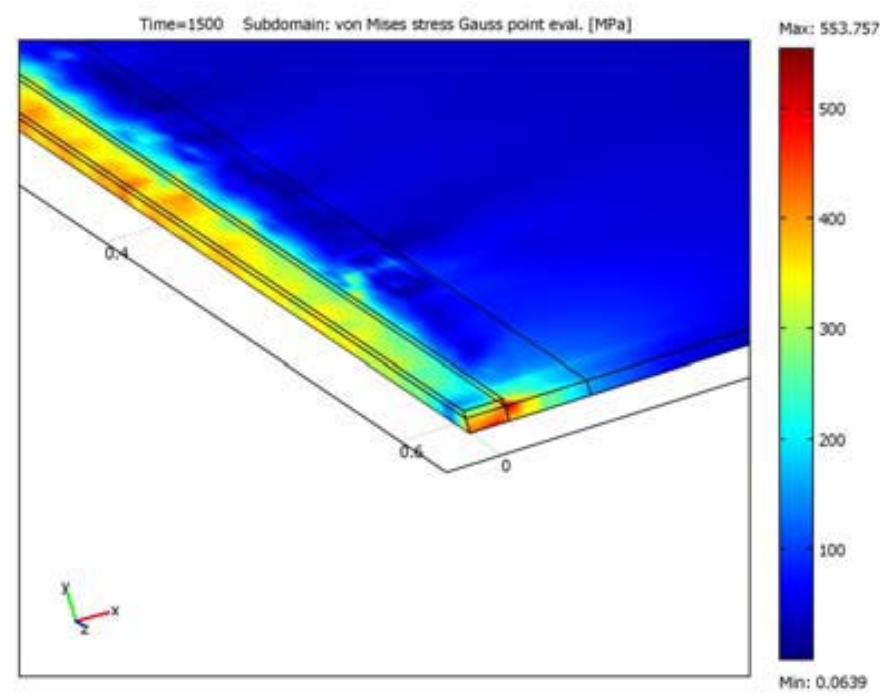

Fig. 3. Von Mises stresses when the system is cooled down. The high stresses on the corner are due to boundary effects

In order to evaluate mechanical toughness and resilience, we assumed that nano-features affect the functioning of the proposed device, but its interactions with the "surrounding world" are scaled into micro-dimensions. We assumed to analyze a PDMS microcantilever. Moreover, when implementing surface stress in $\mathrm{Comsol}^{\circledR}$, one have to consider that it modifies stress-strain relationship in proximity of the surfaces of a body [12]. Therefore, there are two basic actions to do: 1) subdivide the model in two parts (a "thin" surface layer and the bulk); 2) assign an initial stress to the surface layer. The latter comes from the Goldak's equation based analysis. It is done in order to assign a finer mesh to surface. Surface stress is defined in $\mathrm{N} / \mathrm{m}$ since it is a surface related quantity. Anyway, a real surface is not bidimensional but extends inside a body for some atomic layers: a surface has a thickness $h_{\text {surf }}$, then surface stress can be considered as a "bulk" stress $\delta\left(\mathrm{N} / \mathrm{m}^{2}\right)$ acting in a thin layer so that $s=\delta \cdot h_{\text {surf }}$ [12]. It is possible to completely describe the strain conditions at a point with the deformation components - $\left(d_{u}\right.$, $\left.d_{v}, d_{w}\right)$ in $3 \mathrm{D}$ - and their derivatives. You can express the shear strain in a tensor form, $s_{x y}, s_{y z}, s_{x z}$. Following the smalldisplacement assumption, the normal strain components and the shear strain components are given from the deformation as follows: 

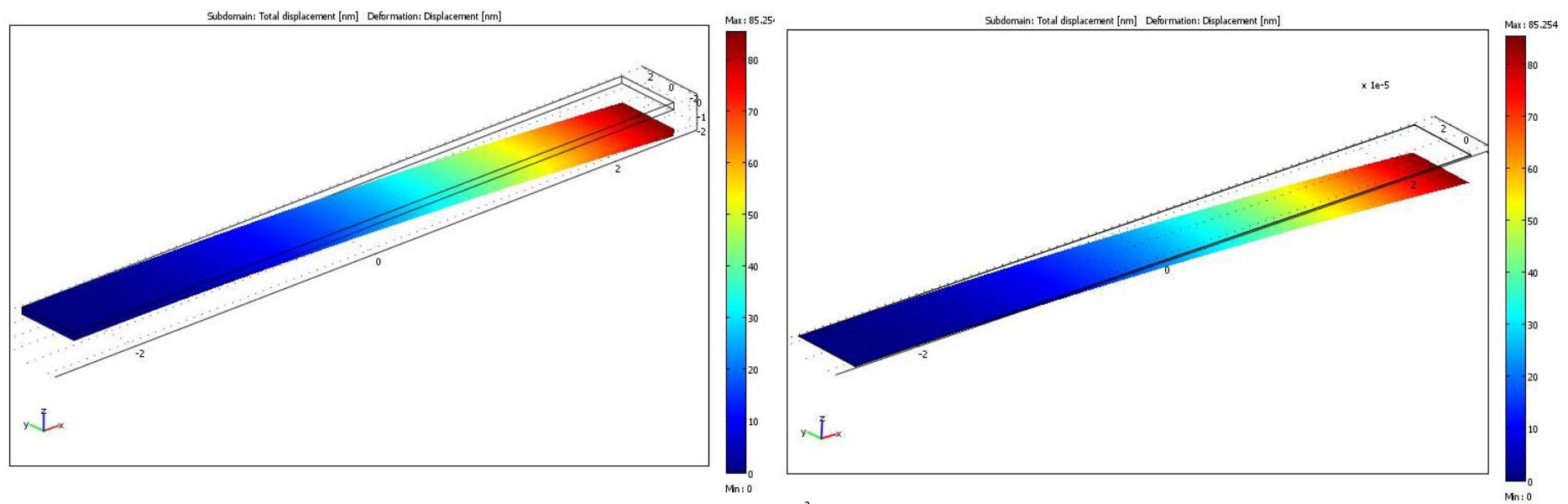

Fig. 4. Deformation displacement for a) bulk and b) surface of a 500x50x5 $\mu \mathrm{m}^{3}$ simulated microcantilever, with $\mathrm{s}=-0.5 \mathrm{~N} / \mathrm{m}$. Cantilever dimensions and surface stress have typical values found in literature.

$$
\begin{aligned}
& s_{x}=\frac{\partial d_{x}}{\partial x} \quad s_{x y}=\frac{1}{2}\left(\frac{\partial d_{x}}{\partial y}+\frac{\partial d_{y}}{\partial x}\right) \\
& s_{y}=\frac{\partial d_{y}}{\partial y} \quad s_{y z}=\frac{1}{2}\left(\frac{\partial d_{y}}{\partial z}+\frac{\partial d_{z}}{\partial y}\right) \\
& s_{z}=\frac{\partial d_{z}}{\partial z} \quad s_{x z}=\frac{1}{2}\left(\frac{\partial d_{x}}{\partial z}+\frac{\partial d_{z}}{\partial x}\right)
\end{aligned}
$$

The symmetric strain tensor $\mathbf{s}$ consists of both normal and shear strain components $\mathbf{s}$, whilst the stress in a material is described by the symmetric stress tensor $\boldsymbol{\delta}$ :

$$
\mathbf{S}=\left[\begin{array}{ccc}
S_{x} & S_{x y} & S_{x z} \\
S_{x y} & S_{y} & s_{y z} \\
S_{x z} & S_{y z} & S_{z}
\end{array}\right] ; \boldsymbol{\delta}=\left[\begin{array}{lll}
\delta_{x} & \tau_{x y} & \tau_{x z} \\
\tau_{x y} & \delta_{y} & \tau_{y z} \\
\tau_{x z} & \tau_{y z} & \delta_{z}
\end{array}\right]
$$

Stress tensor consists of three normal stresses $\left(\delta_{x}, \delta_{y}, \delta_{z}\right)$ and six, or if symmetry is used, three shear stresses $\left(\tau_{x y}, \tau_{y z}, \tau_{x z}\right)$. We based implementation of the structural mechanics model on a weak formulation of the equilibrium equations expressed in the global stress components.

$$
\begin{aligned}
& -\frac{\partial \delta_{x}}{\partial x}-\frac{\partial \tau_{x y}}{\partial y}-\frac{\partial \tau_{x z}}{\partial z}=F_{x} \quad-\frac{\partial \tau_{x y}}{\partial x}-\frac{\partial \delta_{y}}{\partial y}-\frac{\partial \tau_{y z}}{\partial z}=F_{y} \\
& -\frac{\partial \tau_{x z}}{\partial x}-\frac{\partial \tau_{y z}}{\partial y}-\frac{\partial \delta_{z}}{\partial z}=F_{z}
\end{aligned}
$$

where $\mathbf{F}$ denotes the volume forces (body forces). Using compact notation, you can write this relationship as $-\nabla \cdot \boldsymbol{\delta}=\mathbf{F}$, where $\boldsymbol{\delta}$ is the stress tensor. Substituting the stress-strain and strain-displacement relationships in the above equation results in Navier's equation expressed in the displacement. Toughness and resilience were inspected by

means of the same model, but considering a static and transient analysis, respectively. For static conditions including temperature, Navier's equation reads $-\nabla \bullet(c \nabla \mathbf{u})=\mathbf{F}$; on the other hand, a transient problem requires the introduction of Newton's Second Law:

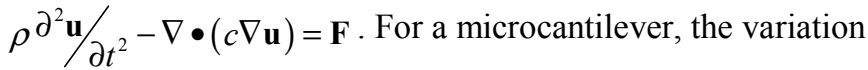
of its surface stress corresponding to one-unit deflection can be expressed by Stoney's formula as follows [13]:

$$
\frac{\Delta \sigma}{\Delta z}=\frac{E h^{2}}{4 L^{2}(1-v)}
$$

where $\Delta \delta$ represents the variation of surface stress; $\Delta z, h$ and $L$ represent the variation of deflection, the thickness and length of the microcantilever, respectively (in nanometer). Elastic Young modulus $E$ and Poisson's ratio $v$ of the microcantilever have been set according to the PDMS properties [14]. A reduction of $h$ or an increase of $L$ leads to a decrease of $\Delta \delta / \Delta z$, i.e., an improvement of sensitivity, at the cost of degrading stability. A number of simulations have been carried out, applying different values of force/area and force/volume as boundary and volume constraints, respectively. Retrieved results have been useful to understand the operative limits of the pressure sensor, and let us affirm its robustness for practical applications. Fig. 4 depicts some results, but more details about numerical simulations will be given in an extended version of this draft. The cantilever sensor, in PDMS, is even covered, that is coated, by a stratum of packaging in PDMS with different quality. Other authors use as coating a PDMS-coated microresonator to allow clear transition, hence a variation of temperature related to tissue touching. Some comments about results proposed in Fig. 4: the system bends downward since the initial stress is negative (i.e. compressive) and located in proximity of the upper surface of the beam. The compressive initial stress in fact forces the surface to expand and so generates a bending moment. The two layers are linked together, then their 
displacements are the same: the free end value is about -85 $\mathrm{nm}$.

After these simulations, we approached the problem of coupling between the deformations and the electric field and how it affects the stability and impedance of the device. Our model solves the electrostatic equation in the air domain surrounding the beam using the Arbitrary LagrangianEulerian (ALE) method to account for geometry changes associated with the deformation. We simulated a cantilever beam having length $300 \mu \mathrm{m}$; width $20 \mu \mathrm{m}$; and thickness 2 $\mu \mathrm{m}$. The cantilever beam, made of PDMS, is fixed at one end but is otherwise free to move. It is coated with a thin conductive layer from the lower side. The packaging has been represented as an air-filled chamber that is electrically insulated, in which the beam resides. However, the lower side of the chamber has a grounded electrode. An electrostatic force caused by an applied potential difference between the two electrodes bends the beam toward the grounded layer below it. To compute the electrostatic force, we calculated the electric field in the surrounding air. The model considers a layer of air $20 \mu \mathrm{m}$ thick both above and to the sides of the beam, and the air gap between the bottom of the beam and the grounded layer is initially $2 \mu \mathrm{m}$. As the beam bends, the geometry of the air changes continuously. Using the ALE method, the model takes this displacement into account when computing the potential field. When the geometry deforms, the electric field between the electrodes continuously changes as a result of the bending. We implemented a $2 \mathrm{D}$ and a $3 \mathrm{D}$ version of the model. In the latter, we exploited symmetry on the zx-plane at $y=0$. The electrostatic field in the air and in the beam is governed by the electrostatic equation $-\nabla \cdot(\varepsilon \nabla V)=0$ where derivatives are taken with respect to the spatial coordinates. These are independent of the deformation of the material. The numerical model, however, needs to represent the electric potential and its derivatives on a mesh which is moving with respect to the spatial frame

The necessary transformations are taken care of by the ALE application mode, which also contains smoothing equations governing the movement of the mesh in the air domain. The lower boundary of the cantilever connects to a voltage source with a specified bias potential, $V_{i n}$. The bottom of the chamber is grounded, while all other boundaries are electrically insulated. Weak constraints make it possible to evaluate the total surface charge, $Q$, on the beam electrode with high accuracy, something which is necessary for calculating the capacitance of the system. The force density that acts on the electrode of the beam results from Maxwell's stress tensor:

$$
\mathbf{F}_{e s}=-\frac{1}{2}(\mathbf{E} \cdot \mathbf{D}) \mathbf{n}+(\mathbf{n} \cdot \mathbf{E}) \mathbf{D}^{T}
$$

where $\mathbf{E}$ and $\mathbf{D}$ are the electric field and electric displacement vectors, respectively, and $\mathbf{n}$ is the outward normal vector of the boundary. This force is always oriented along the normal of the boundary. Navier's equations which govern the deformation of a solid are more conveniently written in a coordinate system which follows and deforms with the material. In this case, these reference or material coordinates are identical to the actual mesh coordinates. Therefore, the solid equations are not affected by the use of ALE for the electrostatic field.

This 2D model solves the structural deformation of the beam using the plane strain approximation. A plane stress assumption is probably closer to reality, but would not be consistent with the $2 \mathrm{D}$ electrostatics formulation which is intrinsically of the same kind as the structural plane strain equations.

Because the electrostatic field in and the structural deformations are tightly coupled in this model, the capacitance of the system depends very much on its structural properties. Here, it is necessary to differentiate between the incremental DC capacitance $C_{D C}$ and the frequencydependent AC capacitance $C_{A C}$. The former is defined as the change in surface charge, $\Delta Q$, induced by a step change in the applied voltage from $V_{i n}=V_{0}$ to $V_{i n}=V_{0}+\Delta V_{i n}$. On the other hand, $C_{A C}$ can be easily obtained as the ratio between the complex-valued surface charge $Q_{A C}$ and the amplitude of a small sinusoidal signal $V_{A C}$, of which $Q_{A C}$ is the response.

A positive feedback exists between the electrostatic forces and the deformation of the cantilever beam. The forces bend the beam and thereby reduce the gap to the grounded substrate. This action, in turn, increases the forces. At a certain voltage the electrostatic forces overcome the stress forces, the system becomes unstable, and the gap collapses. This critical voltage is called the pull-in voltage. At applied voltages lower than the pull-in voltage, the beam stays in an equilibrium position where the stress forces balance the electrostatic forces. Fig. 5 shows the steady state solution for the 3D case. Fig. 6 shows the shape of the cantilever's deformation extracted from 3D results along the long edge for different applied potential values.

When solving for a level higher than the pull-in voltage, the solution ceases to converge before the beam touches the substrate. This is an effect of the ALE method not being able to handle topology changes. By scanning over different applied voltages and using the parametric solver, you can study the beam's behaviour and estimate the pull-in voltage.

Fig. 7 shows the deflection of the beam's end in a 2D model for different applied potential values. This latter figure indicates that the pull-in voltage of the $300 \mu \mathrm{m}$ long cantilever beam is somewhere between $6.3 \mathrm{~V}$ and $6.4 \mathrm{~V}$. Using a finer mesh and computing the solution for a number of voltages in this range should reduce the size of this interval. The deformation of the $3 \mathrm{D}$ model with applied potential $6.1 \mathrm{~V}$ is roughly the same as for the $2 \mathrm{D}$ model with the larger applied potential, which suggests that the pull-in should appear at roughly between $6.1 \mathrm{~V}$ and $6.2 \mathrm{~V}$. For comparison, computations in [15] lead to the pull-in voltage $V_{P I}$ which is equal to $6.35 \mathrm{~V}$ if using an infinite width in order to disable the fringing-field correction, or results in $V_{P I}=6.07$ $\mathrm{V}$ by setting a $20 \mu \mathrm{m}$-width microcantilever beam. 


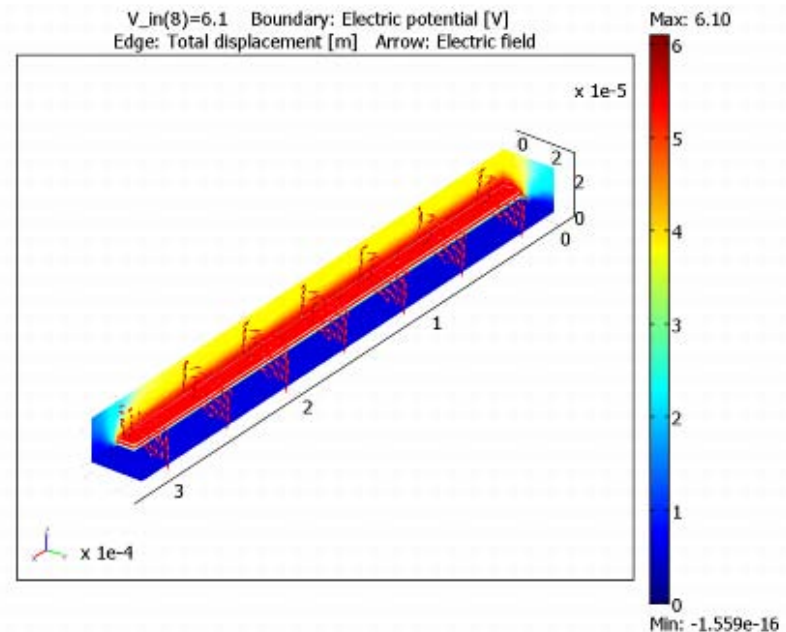

Fig. 5. (3D model) Steady-state solution for the 3D cantilever-beam model with an applied potential of $6.1 \mathrm{~V}$. The boundaries display the electric potential; the arrows show the electric field; the black edges indicate the nondeformed beam geometry; and the white edges delineate the deformed geometry.

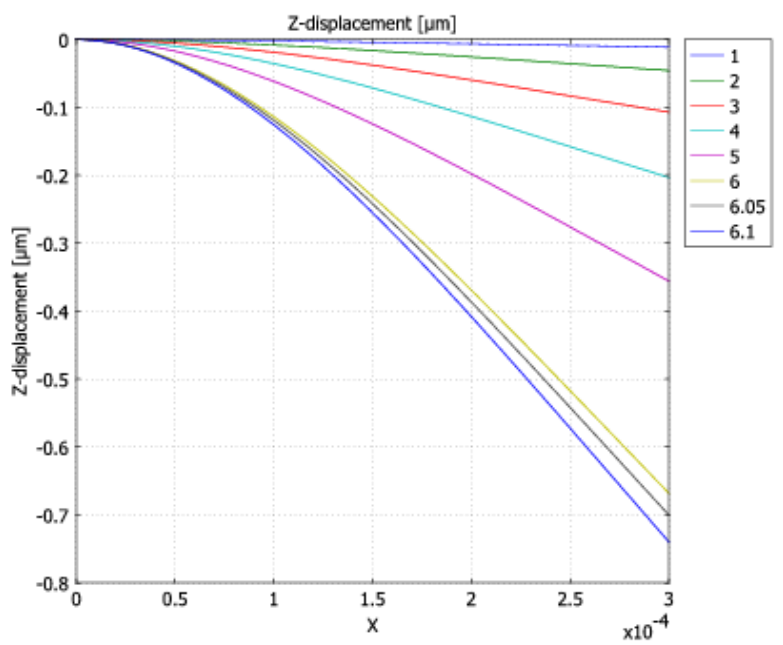

Fig. 6. (3D model) The deformed shape of the cantilever along the long edge for several applied potential values between $1 \mathrm{~V}$. and $6.1 \mathrm{~V}$.

A simple finite difference approximation solves and calculates the DC capacitance, which increases with increasing bias (Fig. 8). On one hand, this is consistent with the behaviour of an ideal parallel plate capacitor, whose capacitance increases with decreasing distance between the plates. But this does not explain all of the increase. In fact, most of it is due to the gradual softening of the coupled electro-mechanical system. This effect leads to a larger structural response for a given voltage increment at higher bias, which in turn means that more charge must be added to retain the voltage difference between the electrodes.

The AC capacitance evaluated at $6.3 \mathrm{~V}$ bias shows a typical behaviour (Fig. 9). Below the fundamental resonance, the capacitance increases with frequency because there is increasing positive feedback between the voltage and the displacement. Above the resonance, the beam vibrates out-ofphase with the electrical forces acting on it, leading to negative capacitance for frequencies where the displacement is large. In the low frequency end, the AC capacitance converges towards the DC capacitance.

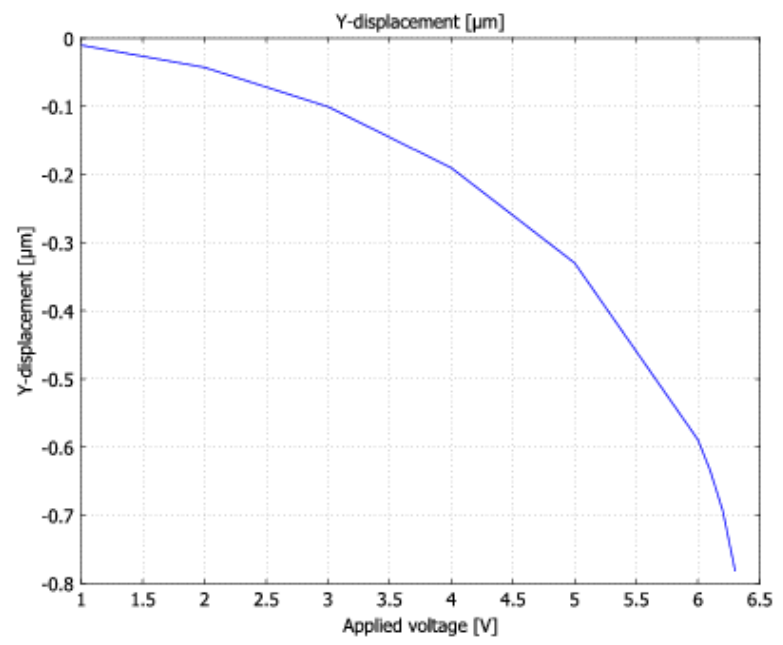

Fig. 7. (2D model) Vertical displacement at the beam tip as a function of the applied voltage. The solution ceases to converge just before the beam touches the substrate.

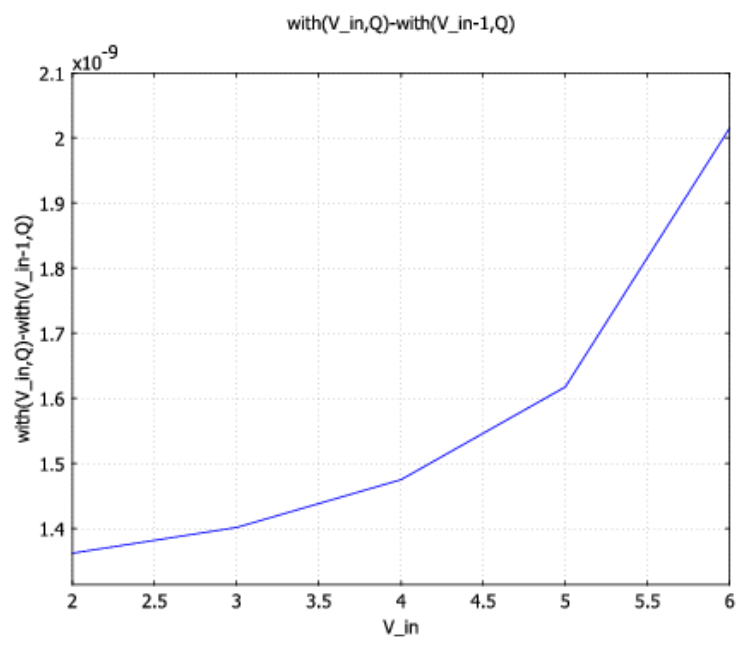

Fig. 8. Estimation of the DC capacitance as a quotient between consecutive increases in surface charge and applied voltage.

Note that the fundamental frequency decreases with increasing bias. At $6.3 \mathrm{~V}$, the frequency has decreased to less than half its unbiased value of about $29 \mathrm{kHz}$. While the nonconvergence of the nonlinear solver at and above the pull-in voltage can be explained from the physical point of view as a pull-in or collapse, and from the numerical view point can be blamed on the ALE method's inability to handle topological changes, the pure mathematical explanation is that at pull-in, the fundamental eigenfrequency approaches zero.

Finally, we evaluated the ability of preventing corrosion of the sensor packaging by analyzing the leakage currents of our prototype [16]. In this context, the temperature of the fluid and the characteristic of the contact between fluid and encapsulation are the key point of the study. 
$Q$

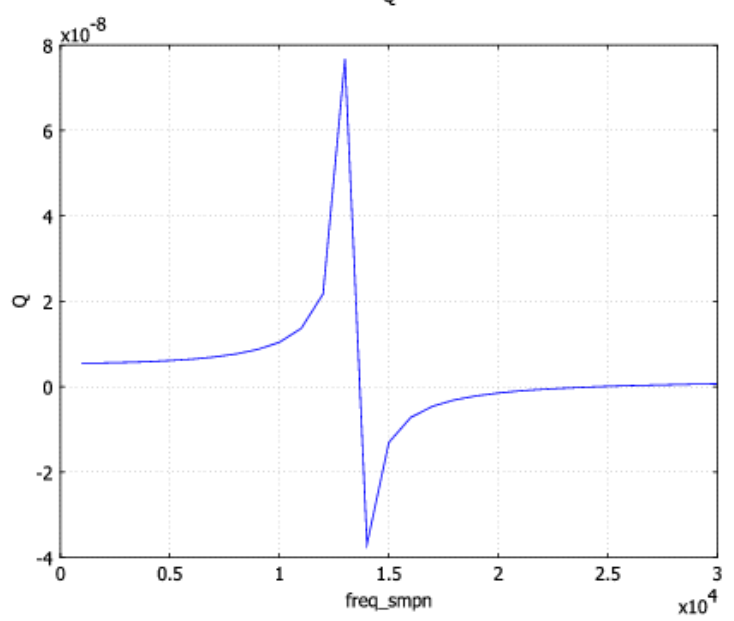

Fig. 9. The AC capacitance of the $2 \mathrm{D}$ cantilever beam reaches a maximum at the fundamental resonance frequency of the coupled system, where it also switches sign because of the rapid phase shift in the structural response.

As longer packaging films provide a protection against corrosion caused by physiological solution, as better the packaging performs electrically insulating encapsulation. Therefore, we planned to save PDMS microcantilever in a biocompatible packaging, with exposed superior part. Leakage currents were estimated by modelling the PDMS sensors as immerged within a saline solution, using timedependent governing equation (refer to [17] for details) in order to verify the insulation during a number of months. For leakage current test, the geometry was biased with a $5 \mathrm{~V}_{\mathrm{dc}}$ continuously, and capacitive abilities of the packaged microcantilever have been numerically measured in order to verify the amount of leakage currents as a function of days. Obtained results are encouraging, and will be deeply discussed in the full version of the paper.

\section{CONCLUSIONS}

A preliminary stress analysis of a packaging of a PDMS cantilever sensor for biomedical applications is presented in this paper. The sensor and its packaging are suitable for specific issues where blood pressure and breathing problems can provoke damages if tissues are touched in a wrong way; for instance, cells disorders due to cancer, aneurism in aorta or in brain, removing materials from lungs in case of pulmonary cancer. For the aforementioned taxonomy, pressure plays a key issue. Particularly for lung cancer where adhesions are investigated, the impact of packing on touching by means of feel pin is of major consequences due to breathing variation. The pressure is transduced in a current that allows the surgeons in navigation and to calibrate the investigation.

\section{ACKNOWLEDGEMENT}

This paper, included in a wide international project, is supported by the Italian-Croatian scientific agreement according to the Approval Letter prot.269/140305 of the Italian Foreign Ministry.

\section{REFERENCES}

[1] Harrison R.R. "The Design of Integrated Circuits to Observe Brain Activity", Proceedings of the IEEE, vol. 96, n. 7, 2008.

[2] Zhang X., Pei W., Gui Q., Chen H., "Low Power Integrated Circuits for Wireless Neural Recording Applications". IEEE Asia Pacific Conference on Circuits and Systems, pp. 650-653, 2008.

[3] Yuce M.R., Wentai L., Moo Sung Chae, Jung Suk Kim, “A Wideband Telemetry Unit for Multi-Channel Neural Recording Systems", IEEE International Conference on Ultra-Wideband, pp. 612-617, 2007.

[4] Sodagar A.M., Wise K.D., Najafi, K., "A Fully Integrated MixedSignal Neural Processor for Implantable Multichannel Cortical Recording", IEEE Transactions on Biomedical Engineering, vol. 54, n. 6, pp. 1075-1088, 2007.

[5] Azin M., Mohseni P., "A 94- $\mu$ W 10-b Neural Recording Front- End for an Implantable Brain-Machine-Brain Interface Device", Biomedical Circuits and Systems Conference, pp. 221-224, 2008.

[6] Aziz J.N.Y., Abdelhalim K., Shulyzki R., Genov R., Bardakjian B.L., Derchansky M., Serletis D., Carlen P.L. "256-Channel Neural Recording and Delta Compression Microsystem With 3D Electrodes", IEEE Journal of Solid-State Circuits, vol. 44, n. 3, pp. 995-1005, 2009.

[7] Boretos J.W., "Considerations on encapsulation for acute/chronic longevity of electronic implants", in Synthetic Biomedical Polymers, M. Szycher and W. J. Robinson, Eds. Westport, CT: Technomic, 1980, pp. 187-200.

[8] Jeutter D.C., "Biomedical telemetry techniques", CRC Critical Rev. Biomed. Eng., vol. 8, pp. 121-174, 1982.

[9] Donaldson P.E.K., "The encapsulation of microelectronic devices for long-term surgical implantation", IEEE Trans. Biomed. Eng., vol. 23, pp. 281-285, 1976.

[10] Cai D.K., Neyer A., Kuckuk R., Heise H.M., "Optical absorption in transparent PDMS materials applied for multimode waveguides fabrication", Optical Material, vol. 30, pp. 1157-1161, 2008.

[11] Wikander L., Karlsson L., Mäsström M. and Webster P., "Finite Element Simulation and Measurement of Welding Residual Stresses", Modelling and Simulation in Materials Science and Engineering, vol. 2, n. 4, pp. 845-864, 1994.

[12] Sader J.E., "Surface stress induced deflections of cantilever plates with applications to the atomic force microscope: Rectangular plates", J. Appl. Phys., vol. 89, pp. 2911-2921, 2001

[13] Tsui Y. C. and Clyne T. W., "An analytical model for predicting residual stresses in progressively deposited coating, Part 1: Planar geometry," Thin Solid Films, vol. 306, pp. 23-33, 1997.

[14] Akogwu O., Kwabi D., Munhutu A., Tong T., Soboyejo, W. O., "Adhesion and cyclic stretching of Au thin film on poly(dimethylsiloxane) for stretchable electronics", Journal of Applied Physics, vol. 108, n. 12, pp. 123509-123509-6, 2010.

[15] Gupta R.K., Electrostatic Pull-In Structure Design for In-Situ Mechanical Property Measurements of Microelectromechanical Systems (MEMS), Ph.D. thesis, MIT, 1997.

[16] Hsu J.-M., Rieth L., Normann R.A., Tathireddy P., Solzbacher F., "Encapsulation of an Integrated Neural Interface Device With Parylene C", IEEE Transactions on Biomedical Engineering, vol. 56, n. 1, pp. 23-29, 2009.

[17] Persano A., Quaranta F., Cola A., et al., "Ta2O5 Thin Films for Capacitive RF MEMS Switches," Journal of Sensors, vol. 2010, Article ID 487061, 5 pages, 2010. doi:10.1155/2010/487061 